\title{
Analytical Evaluation of the Ratio Between Injection and Space-Charge Limited Currents in Single Carrier Organic Diodes
}

\author{
Ángel Luis Alvarez, Member, IEEE, Belén Arredondo, Beatriz Romero, Xabier Quintana, \\ Araceli Gutiérrez-Llorente, Ricardo Mallavia, and José Manuel Otón
}

\begin{abstract}
An analytical, complete framework to describe the current-voltage $(I-V)$ characteristics of organic diodes without the use of previous approaches, such as injection or bulk-limited conduction is proposed. Analytical expressions to quantify the ratio between injection and space-charge-limited current from experimental $I-V$ characteristics in organic diodes have been derived. These are used to propose a numerical model in which both bulk transport and injection mechanisms are considered simultaneously. This procedure leads to a significant reduction in computing time with respect to previous rigorous numerical models. In order to test the model, different diode structures based on two different polymers: poly(2-methoxy-5- $\left\{3^{\prime}, 7^{\prime}\right.$-dimethyloctyloxy $\}-p$ phenylenevinylene) (MDMO-PPV) and a derivative of the poly (2,7fluorene phenylidene) [PFP: $\left.(\mathrm{CN})_{2}\right]$, have been fabricated. The present model is excellently fitted to experimental curves and yields the microscopic parameters that characterize the active layer.
\end{abstract}

Index Terms-Hopping transport, injection current, polymer light-emitting diode (PLED), space charge.

\section{INTRODUCTION}

$\mathbf{E}$ LECTRICAL behavior of polymer light-emitting diodes (PLEDs) has been generally interpreted by a paradigm consisting of two processes: carrier injection at the metalorganic interface and subsequent carrier transport along the bulk. In terms of an equivalent circuit, the process may be understood as an electric-field-controlled current source, in series with a variable resistance, dependent on the injected space charge.

In general, current density-voltage characteristics $\left(J_{b}-V_{b}\right)$ are modeled assuming simplifications, such as injection-limited or bulk-limited conduction. However, for the typical active layers

Manuscript received May 22, 2007, revised October 24, 2007. This work was supported in part by the Universidad Rey Juan Carlos, in part by the Comunidad Autónoma de Madrid under Projects URJC-TIC-044-1 and S0505/ESP/000417, and also in part by the Spanish Ministry of Education and Science under Project TEC2006-13392-C02-02/MIC. The review of this brief was arranged by Editor J. Kanicki.

Á. L. Álvarez, B. Arredondo, B. Romero, and A. Gutiérrez are with the Dpt. Ingeniería Telemática y Tecnología Electrónica, Universidad Rey Juan Carlos, Edif. Departamental II, 28933 Madrid, Spain (e-mail: angelluis.alvarez@urjc.es; belen.arredondo@urjc.es; beatriz.romero@urjc.es; araceli.gutierrez@urjc.es).

X. Quintana and J. M. Otón are with the Depto. Tecnología Fotónica, E.T.S.I. Telecomunicación, Ciudad Universitaria, 28040 Madrid, Spain (e-mail: xabi@tfo.upm.es; jmoton@tfo.upm.es).

R. Mallavia with the Instituto de Biología Molecular y Celular, Universidad Miguel Hernández, Edificio Torregaitán, Elche 03202 Alicante, Spain (e-mail: r.mallavia@umh.es).

Color versions of one or more of the figures in this brief are available online at http://ieeexplore.ieee.org.

Digital Object Identifier 10.1109/TED.2007.912995 used in organic diodes, both processes may limit conduction in a comparable way when a moderate barrier for carrier injection is present, e.g., $0.2-0.5 \mathrm{eV}$. In this case, $J_{b}-V_{b}$ characteristics need to be calculated by complex numerical models that require difficult and time consuming procedures [1]-[3]. In this paper, we establish an analytical framework to solve not only the injection and bulk limited cases, but also devices where both mechanisms play a significant role. Moreover, we define a simple analytical expression to quantify the ratio between both limiting conduction regimes, injection and bulk, for any measured $J_{b}-V_{b}$ characteristic. Guidelines to derive an analytical function for the electric field at the interface between metal and polymer are also given. Some of the resulting expressions may be readily used to model the single-carrier organic diode response with a significant computing time reduction.

Proposed injection mechanisms in literature have been, so far, based either on a classical approach, e.g., the thermionic effect, where a Richardson-like constant needs to be defined [4], [5], or on a microscopic approach of carrier hopping between discrete states [6]. Within the latter approach, an analytical model for the injection current density, $J_{\mathrm{inj}}$, including both structural and energetic disorder was proposed in [7]. Experiments aimed to check the validity of both approaches have been performed measuring the temperature dependence of the injected current [8]. Attending to these studies, the hopping nature of both charge transport and injection from the contact into localized states of the polymer is currently well established, and will constitute our framework.

All mentioned injection mechanisms are dependent on electric-field, $J_{\mathrm{inj}}\left(E_{T}\right)$, where $E_{T}$ is the total electric field, and include a slight modulation of the effective barrier height due to the image-charge effect. Therefore, an accurate knowledge of the electric field at the interface, $E_{T}(x=0)$ is necessary for a reliable evaluation of the injected current. This is not, in general, an easy task.

The other process regarding carrier transport in the bulk is usually formulated in terms of the Mott-Gurney formalism [9], which is derived from three fundamental equations

$$
\begin{aligned}
J_{b} & =e \mu\left[p_{n}\right] E_{\mathrm{T}}(x) \\
\frac{d E_{T}(x)}{d x} & =\frac{e[p]}{\varepsilon \varepsilon_{o}} \\
V_{b} & =\int_{0}^{t} E_{T}(x) d x
\end{aligned}
$$


where $J_{b}$ is the total current density in the bulk, $E_{T}(x)$ the position-dependent electric field, $e$ the elementary charge, $\mu$ the equilibrium carrier mobility, $\varepsilon_{0}$ the vacuum permittivity, $\varepsilon$ the material dielectric constant, $t$ the active layer thickness, $V_{b}$ the external bias (after the effect of the built-in potential resulting from the asymmetric work functions of the electrodes is corrected), $[p]$ the total carrier density, and $\left[p_{n}\right]$ the density of carriers contributing to transport. Common conjugated polymers employed in organic light-emitting diodes, exhibit large bandgaps and are not deliberately doped during synthesis. Thus, the existence of a neutralized free charge $\left[p_{0}\right]$ associated to shallow ionized impurities, is generally discarded, and it is assumed that the total carrier density $[p]$ arises from the injected charge. Despite $\left[p_{0}\right]$ being neglected, it is generally accepted that $[p] \neq\left[p_{n}\right]$ due to the presence of carrier traps. Even in the case that trapping effect is discarded, Arkhipov et al. established that not all the injected charge $[p]$ contributes to transport in a disordered semiconductor. They base their study on the approximated concept of transport energy level, $E_{\mathrm{tr}}$. This is a statistical tool that simplifies pure hopping transport in a positionally and energetically disordered medium, and approximates it to a trap-controlled effective transport [10]. Thus, carrier density contributing to transport is always a fraction of the total injected charge. In fact, $p\left(p_{n}\right)$ is a nonlinear function that has been analytically formulated in [11]. It depends, among other factors, on a complex interplay between the energy density of states and the level of injected carriers. However, assuming that carrier densities are not excessively high, in a range that may cover many of the typical currents in organic devices, a quasi-linear relation between $\left[p_{n}\right]$ and $[p]$ may be established [12]

$$
\left[p_{n}\right]=\Phi[p] \quad \text { with } \Phi=\frac{\tau_{o} \nu_{o} N_{t}}{\int_{-\infty}^{E_{t r}} g(E) \exp \left(\left(E_{\mathrm{tr}}-E\right) / k T\right) d E}
$$

where $N_{t}$ is the total site density, $\nu_{\mathrm{O}}$ the attempt-to-jump frequency, $\tau_{\circ}$ the carrier lifetime in the transport level, and $\mathrm{g}(E)$ the density of states (DOS).

On the other hand, either in the referred Mott-Gurney formalism or in those more sophisticated [9], [13], the dependence of mobility on the electric field and space charge is usually not considered in the first approximation. A similar approach is assumed in our model, since otherwise, an analytical solution is not feasible. Consequences of this simplification are briefly discussed in Section IV.

Solutions of the electric field function, $E_{T}(x)$, from (1)(3), exhibit very different spatial dependences in two extreme cases: when significant injected charge is present via ohmic contact, or when the injection barrier is high enough to consider injected current negligible, as in the case of a capacitor. In the latter case, the internal electric field must be uniform, it being $E_{T}(0)=V_{b} / t$ at the interface, while in the former case, a dependence on the square root of the spatial coordinate is predicted by (1), giving $E_{T}(0)=0$ [9], [13]. Then, it may be considered that the effective field at the interface $E_{T}(0)$ falls within both limits $\left(0-V_{b} / t\right)$, when a moderate injection barrier from metal to organic is present. In this work we propose an easy formalism to determine this field, and therefore, to obtain the $J_{b}-V_{b}$ characteristic of single carrier organic diodes without assuming previous approaches concerning injection or spacecharge-limited transport.

\section{THEORY}

For that purpose, we invoke the superposition principle to split the total $E_{T}(x)$ in two components, $E_{T}(x)=E_{\mathrm{sc}}(x)+E_{0}$. Where $E_{\mathrm{sc}}(x)$ is due to the space charge effect, and $E_{0}$ is the remaining uniform field in the bulk, that must verify

$$
E_{o}=\frac{V_{b}-\int_{0}^{t} E_{s c}(x) d x}{t} .
$$

Since $E_{0}$ is deduced in terms of the voltage drop along the bulk, the contribution from the image charge effect, which is confined to the very thin region close to the interface, may be neglected in sufficiently thick diodes.

Substituting the value of $E_{T}(x)$ into (1) and combining with (2) and (4), the following first-order, non-linear differential equation for $E_{\mathrm{sc}}(x)$ is obtained:

$$
\frac{J_{b}}{\mu \varepsilon \varepsilon_{o} \Phi}=\frac{d E_{\mathrm{sc}}}{d x}\left(E_{\mathrm{sc}}+E_{o}\right) \text {. }
$$

Applying the usual boundary condition for the space charge field at the interface $E_{\mathrm{sc}}(0)=0$, we obtain

$$
E_{\mathrm{sc}}(x)=-E_{0}+\sqrt{E_{0}^{2}+\frac{2 J_{b} \Phi}{\mu \varepsilon \varepsilon_{0}} x} .
$$

By integrating the electric field along the active layer thickness, and equating to the external voltage $V_{b}$ [see (3)], we obtain the following:

$$
\left(E_{0}^{2}+\frac{2 J_{b} \Phi}{\mu \varepsilon \varepsilon_{o}} t\right)^{\frac{3}{2}}=\frac{3 J_{b} \Phi}{\mu \varepsilon \varepsilon_{o}} V_{b}+E_{0}^{3} .
$$

From (8), a characteristic behavior of $J_{b}$ as a function of both the external bias, $\mathrm{V}_{\mathrm{b}}$, and the field at the interface $\mathrm{E}_{0}$, is obtained, in the (9) shown at the bottom of the page.

It is worth noticing that when $\mathrm{E}_{0}$ vanishes, (9) reproduces the Mott-Gurney expression in the presence of carrier trapping

$$
J_{s c}=\frac{9}{8 \Phi} \mu \varepsilon \varepsilon_{o} \frac{V_{b}^{2}}{t^{3}}
$$

If $E_{0}$ equals the other limit value, $V_{b} / t$, we are in a situation similar to a capacitor, where $\mathrm{J}_{\mathrm{b}}=0$. In fact, it may be proven that $\mathrm{E}_{0}$ approaches $\mathrm{V}_{\mathrm{b}} / \mathrm{t}$ asymptotically for injection barrier $(\Delta)$ tending to infinity, and consequently also, $J_{\mathrm{inj}}=0$. In any case, before reaching that limit, some of the approximations carried out, as that of neglecting $\left[\mathrm{p}_{0}\right]$, likely fail.

$$
J_{b}\left(E_{0}, V_{b}\right)=\mu \varepsilon \varepsilon_{0} \frac{\left(9 V_{b}^{2}-12 E_{0}^{2} t^{2}\right)+\sqrt{\left(12 E_{0}^{2} t^{2}-9 V_{b}^{2}\right)^{2}-192 \cdot E_{0}^{3} t^{3}\left(E_{0} t-V_{b}\right)}}{16 \Phi t^{3}}
$$


The combination of any injection model, $\mathrm{J}_{\mathrm{inj}}=\mathrm{J}_{\mathrm{inj}}\left(\mathrm{E}_{0}\right)$, with both (9) and the continuity equation at the interface for the current density under steady regime (in the absence of carrier recombination), provides a complete system that allows to derive a function for the total diode current $\mathrm{J}_{\mathrm{b}}\left(\mathrm{V}_{\mathrm{b}}\right)$. Thus, providing that mobility and physical parameters of $\mathrm{J}_{\text {inj }}$ are known, (11) may be numerically solved for any bias $\mathrm{V}_{\mathrm{b}}$ to determine $\mathrm{E}_{0}$, and hence, the current density $\mathrm{J}_{\mathrm{b}}$. Alternatively, one can readily derive with the aid of computational algebra, either from (8) or (9), an analytical expression for $\mathrm{E}_{0}$ as a function of $\mathrm{J}_{\mathrm{b}}, \mathrm{V}_{\mathrm{b}}$, that may, in turn, be used to eliminate $\mathrm{E}_{0}$ in (11), so obtaining an implicit function for the total diode current $\mathrm{J}_{\mathrm{b}}=\mathrm{f}\left(\mathrm{J}_{\mathrm{b}} \mathrm{V}_{\mathrm{b}}\right)$. The latter procedure has been used in this paper to fit more efficiently our theoretical model to the experimental data, as will be explained further

$$
J_{\text {inj }}\left(E_{0}\right)=J_{b}\left(V_{b}, E_{0}\right) .
$$

It is of major interest to have a J-V expression easily fitted to experimental data. In this sense, our method overcomes the problem of using numerical recipes to determine $\mathrm{E}_{0}$, leading to a significant reduction in computing time by one order of magnitude with respect to rigorous numerical procedures [1].

On the other hand, we can define

$$
\beta=\frac{J_{b}}{J_{\mathrm{sc}}}=\left(\frac{8 \Phi t^{3}}{9 \mu \varepsilon \varepsilon_{0}}\right) \cdot \frac{J_{b}}{V_{b}^{2}}
$$

where $\beta$ is a function varying in the range $[0-1]$, and is useful to reveal the role played by each limiting conduction mechanism, either injection or bulk, in the diode. Thus, for any measured value of $\mathrm{J}_{\mathrm{b}}$ and $\mathrm{V}_{\mathrm{b}}$, and assuming that material mobility is known, $\beta$ provides a quantitative criterion to quickly check the validity of the injection limited $(\beta \cong 0)$ or bulk-limited $(\beta \cong 1$ ) approximations.

\section{EXPERIMENT}

In order to check the validity of our model, singlecarrier diodes with structure anode/hole injection layer/active layer/cathode were fabricated using two different active layers with several thicknesses: 1) poly(2-methoxy-5- $\left\{3^{\prime}, 7^{\prime}-\right.$ dimethyloctyloxy $\}$-p-phenylenevinylene) (MDMO-PPV), and 2) a ciano-derivative of the poly(2,7-fluorene phenylidene) [PFP: $(\mathrm{CN})_{2}$ ] [14]. The 40-nm-thick hole injection layer is based on poly(3,4-ethylenedioxythiophene)/poly(4-styrenesulfonate) (PEDOT:PSS). This anode interface layer, together with the high injection barrier expected between $\mathrm{Al}$ and the lowest unoccupied molecular orbital (LUMO) of the polymers, support the assumption of hole-only conduction. In any case, the voltage range shown in the $-J-V$ plots is below the onset for electroluminescence (EL) in all devices.

The entire fabrication process is carried out in a clean room (class 10000). Commercial indium-tin-oxide (ITO, thickness = $100 \pm 5 \mathrm{~nm}$ ) coated glass is used as substrate. Prior to film deposition, the substrates went through a typical organic material cleaning process. The final diode passive matrix contains eight diodes with two different radii, 0.5 and $0.75 \mathrm{~mm}$, consisting of a common Al cathode, and an individual ITO anode for each
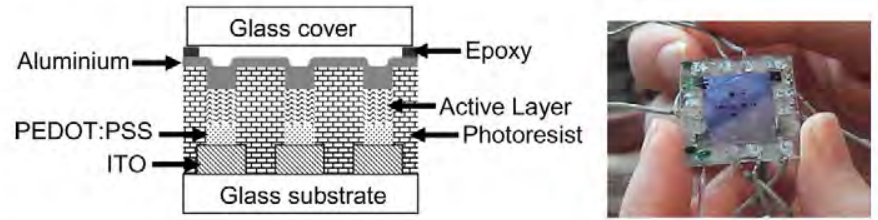

Fig. 1. (a) Layer structure of the fabricated devices. (b) Resulting OLED passive matrix containing eight diodes.

diode. The fabrication process consists of several steps. First, tracks and pads are patterned on the ITO-coated glass by means of a photolithographic process. In a second photolithographic process, photoresist wells are built on top of ITO pads. The insulating behavior of the photoresist avoids leakage currents between adjacent diodes. A hole transport layer of PEDOT/PSS is spin-casted at $6000 \mathrm{r} / \mathrm{m}$ and dried at $110^{\circ} \mathrm{C}$ for $30 \mathrm{~min}$ in $\mathrm{N}_{2}$ atmosphere. Such material is insoluble in organic solvents, and therefore, prevents the photoresist from being redissolved by the polymer solution. The organic active layer is spin-casted at $6000 \mathrm{r} / \mathrm{m}$ and annealed at $115^{\circ} \mathrm{C}$ for $30 \mathrm{~min}$ in $\mathrm{N}_{2}$. The $\mathrm{Al}$ cathode is thermally evaporated on top of the organic layer surface in an atmosphere of $10^{-6}$ torr. Devices are encapsulated using a glass cover attached by a bead of epoxy adhesive [EPOTEK (302-3M)]. This process is carried out into a glove box in inert atmosphere. Fig. 1 (left) shows the layer structure. Finally, contact wires are indium soldered to the ITO pads. The final device is the eight-diode passive matrix, shown in Fig. 1 (right). Biasing is provided through the ITO paths that connect each anode to the outer wire.

The film thickness was measured with an Alpha-Step 200 (Tenkor) contact profilometer. Current-voltage characteristics were recorded using an Agilent $4155 \mathrm{C}$ semiconductor parameter analyzer and an Agilent 41501B SMU pulse generator.

\section{RESULTS}

Spatial dependence of the total electric field $\left(\mathrm{E}_{0}+\mathrm{E}_{\mathrm{sc}}\right)$ obtained with our model, for a 100 -nm-thick sample, $0.5 \mathrm{eV}$ barrier for carrier injection, and biased at $\mathrm{V}_{\mathrm{b}}=20 \mathrm{~V}$, is illustrated in Fig. 2. The total electric field was calculated for a given subset of microscopic parameters, mobility and bias. The calculation of $\mathrm{E}_{0}$ and $\mathrm{E}_{\mathrm{sc}}(\mathrm{x})$ was then carried out separately. First, (11) is solved for the mentioned set of physical parameters, using an injection function $\mathrm{J}_{\mathrm{inj}}\left(\mathrm{E}_{0}\right)$ proposed by Arkhipov et al. [7, eq. (17)]. This is a microscopic mechanism that considers the probability of carrier hopping from the metal to a random positionally and energetically system. Once $\mathrm{E}_{0}$ is known, the electric field due to the space charge was determined using (7).

For high mobility values, the electric field is quasi-uniform across the sample. This intuitively agrees with the fact that a high mobility does not favor carrier accumulation, lowering the space charge effect. However, as mobility decreases, the electric field resembles that of a bulk-controlled mechanism. Notice that the electric field at the interface $(x=0)$ is never higher than 


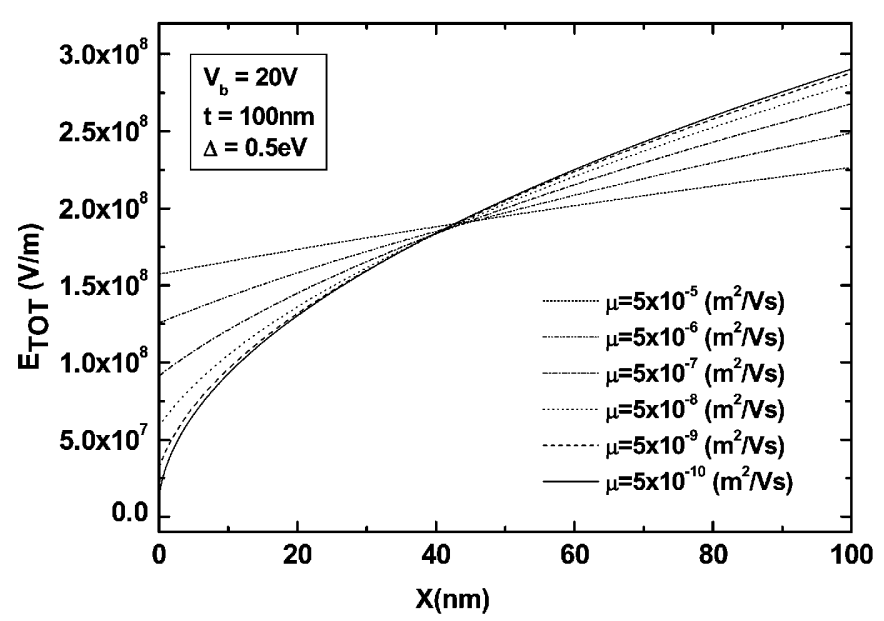

Fig. 2. Spatial distribution of the electric field into the active layer for different mobility values, when diode is biased at $20 \mathrm{~V}$. Structural parameters are: thickness $t=100 \mathrm{~m}$, injection barrier $\Delta=0.5 \mathrm{eV}$. Material parameters are: attempt-to-jump frequency $\nu_{0}=4.75 \times 10^{13} \mathrm{~s}^{-1}$, inverse localization radius $\gamma=5 \times 10^{9} \mathrm{~m}^{-1}$, DOS width $\sigma=50 \mathrm{meV}$, site density $N_{t}=5 \times 10^{20} \mathrm{~cm}^{-3}$, intersite distance $\mathrm{a}=1 \mathrm{~nm}$, and dielectric constant $\varepsilon=4$.

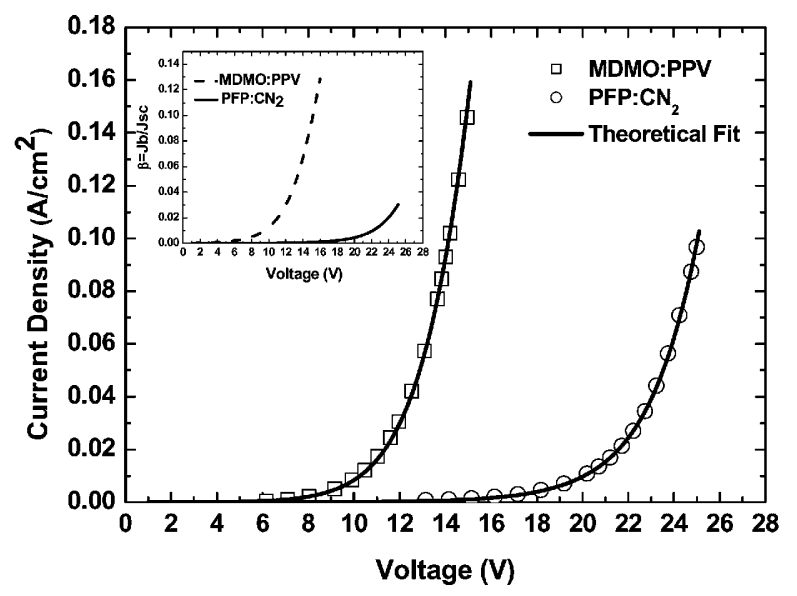

Fig. 3. Experimental $J-V$ curve of PLEDs based on ITO/PEDOT:PSS/ MDMO-PPV/Al (squares), $t$ (MDMO:PPV) $=73 \mathrm{~nm}$, and ITO/PEDOT:PSS/ PFP: $(\mathrm{CN})_{2} / \mathrm{Al}$ (circles) t(PFP: $\left.(\mathrm{CN})_{2}\right)=140 \mathrm{~nm}$, and theoretical fits using the model proposed in this work (solid lines). Parameters obtained from the fit for the MDMO:PPV based PLED are: $\Delta=0.59 \mathrm{eV}, \mu^{*}=8.3 \times 10^{-10} \mathrm{~m}^{2} / \mathrm{V} \cdot \mathrm{s}$, $\sigma=32 \mathrm{meV}, \gamma=4.3 \times 10^{9} \mathrm{~m}^{-1}, \mathrm{a}=0.8 \mathrm{~nm}$, and for the PFP: $(\mathrm{CN})_{2}$ based PLED are: $\Delta=0.68 \mathrm{eV}, \mu^{*}=3.6 \times 10^{-9} \mathrm{~m}^{2} / \mathrm{V} \cdot \mathrm{s}, \sigma=41 \mathrm{meV}, \gamma=3.26 \times$ $10^{9} \mathrm{~m}^{-1}, \mathbf{a}=0.96 \mathrm{~nm}$. Inset shows the variation of $\beta$ with bias in both cases.

$\mathrm{V}_{\mathrm{b}} / \mathrm{t}$. The fact that the electric field may increase over $\mathrm{V}_{\mathrm{b}} / \mathrm{t}$ with increasing layer depth layer is a common feature of the space-charge regime, here observed for $x>50 \mathrm{~nm}$. These results are in perfect agreement with those also shown by Arkhipov et al. [13].

The result of applying our model to the study of PLEDs electrical behavior is presented in Fig. 3. That shows the experimental $\mathrm{J}_{\exp }-\mathrm{V}_{\exp }$ of two diodes, based on the structures: ITO/PEDOT:PSS/MDMO-PPV/Al and ITO/PEDOT:PSS/PFP: $(\mathrm{CN})_{2} / \mathrm{Al}$. The EL onset for both PLEDS was detected above the maximum bias plotted, so that single-carrier conduction is assured. The model proposed in the previous section has been fitted to the experimental data, using the mentioned injection function $\mathrm{J}_{\mathrm{inj}}\left(\mathrm{E}_{0}\right)$ proposed by Arkhipov et al. A number of physical parameters are involved in this model: barrier height for carrier injection $(\Delta)$, energetic width $(\sigma)$ of the DOS for the energy level under Gaussian approximation, carrier inverse localization radius $(\gamma)$, average nearest neighbor distance $(a)$, and the attempt-to-jump frequency $\left(\nu_{0}\right)$. All are well described in [7], and most of them may be physically measured, although not with similar accuracy. Solid lines in Fig. 3 illustrate the fit.

The fitting procedure has been performed using a nondeterministic genetic algorithm, since this type of algorithms is considered more suitable for systems with many parameters [15]. In our case, these parameters are $\mu, \Delta, \sigma, \gamma$, and $a$. Attempt-to-jump frequency $\nu_{\mathrm{o}}$ was previously obtained for each PLED from their EL spectra at high bias. Since it is assumed that $\nu_{0}$ is enhanced by lattice vibrations, spectrally resolved vibronic features allow a good estimation of this parameter, resulting in $\nu_{\mathrm{O}(\mathrm{MDMO}: \mathrm{PPV})}=3.55 \times 10^{13} \mathrm{~s}^{-1}$ and $\nu_{\mathrm{O}(\mathrm{PFP}: \mathrm{CN} 2)}=4.75 \times 10^{13} \mathrm{~s}^{-1}$.

Having an analytical expression for $\mathrm{E}_{0}$ simplifies the fitting method. The experimental data $\mathrm{J}_{\exp }, \mathrm{V}_{\text {exp }}$ together with the initial mobility value (estimated by other physical measurements [16]) are used to evaluate $E_{0}$. Within our model, this is the exact value that should allow $\mathrm{J}_{\mathrm{inj}}$ to verify the continuity equation, so that $\mathrm{J}_{\mathrm{inj}}$ is calculated using $\mathrm{E}_{0}$ and the rest of fitting parameters. The error function between $\mathrm{J}_{\mathrm{inj}}$ and $\mathrm{J}_{\exp }$ is then evaluated, and subsequently, minimized.

Parameters have been actually extracted from the fit to both the experimental $J_{\mathrm{cxp}}-\mathrm{V}_{\mathrm{cxp}}$, and the dimensionless function $\left(\mathrm{dJ}_{\mathrm{cxp}} / \mathrm{dV}_{\mathrm{cxp}}\right) \cdot\left(\mathrm{V}_{\mathrm{exp}} / \mathrm{J}_{\mathrm{exp}}\right)$, which should be independent of pre-factors such as diode radius, attempt-to-jump frequency, etc. For the case of the MDMO:PPV-based PLED the resulting parameters are: $\Delta=0.59 \mathrm{eV}, \mathrm{a}=0.8 \mathrm{~nm}$, $\gamma=4.3 \times 10^{9} \mathrm{~m}^{-1}, \sigma_{(\mathrm{HOMO})}=32 \mathrm{meV}$, and effective mobility $\mu^{*}=8.3 \times 10^{-10} \mathrm{~m}^{2} / \mathrm{V} \cdot \mathrm{s}$. Similarly, parameters for the PFP: $(\mathrm{CN})_{2}$-based PLED are as follows: $\Delta=0.68 \mathrm{eV}, \mathrm{a}=$ $0.96 \mathrm{~nm}, \gamma=3.26 \times 10^{9} \mathrm{~m}^{-1}, \sigma_{(\mathrm{HOMO})}=41 \mathrm{meV}$ and effective hole mobility $\mu^{*}=3.6 \times 10^{-9} \mathrm{~m}^{2} / \mathrm{V} \cdot \mathrm{s}$.

The resulting barrier heights are consistent with data for energy-level alignment between ITO work function and the polymer HOMO levels [14], [17]. $\mu^{*}$ is the mobility corrected by the factor given in (4), taking into account that not all injected charge contributes to transport. Since carrier mobility is known to be dependent on both electric field and charge density [18], [19], these values must be considered an average within the range of applied voltage. Anyway, we notice that, among the fitting parameters, mobility is found to have the lowest influence on the error function. An increase of the error function due to a change in mobility results several orders of magnitude lower than that caused by a similar relative change in barrier or inverse localization radius. Therefore, this approximation is not considered critical for the other parameters. Mobility value for the MDMO:PPV is consistent with that given by [18], [20] for the fitted $\sigma$ value. In turn, that obtained for PFP: $(\mathrm{CN})_{2}$ agrees with typical mobility values for polyfluorenes [21]. The 

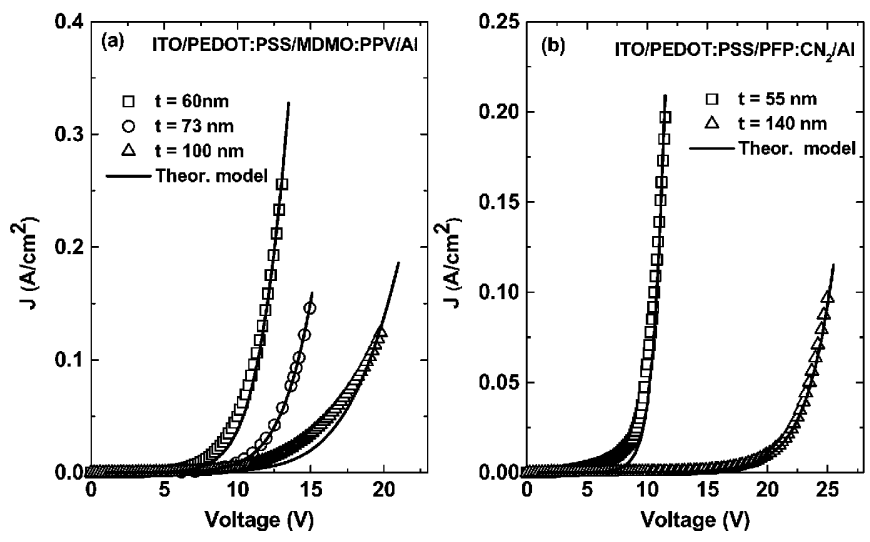

Fig. 4. Experimental $J-V$ characteristics (dotted lines) of devices with identical active layers but different thickness (see legend). Theoretical curves determined with identical parameters at the corresponding thicknesses are plotted as solid lines. (a) MDMO:PPV based diodes. (b) PFP:(CN) $)_{2}$ based diodes.
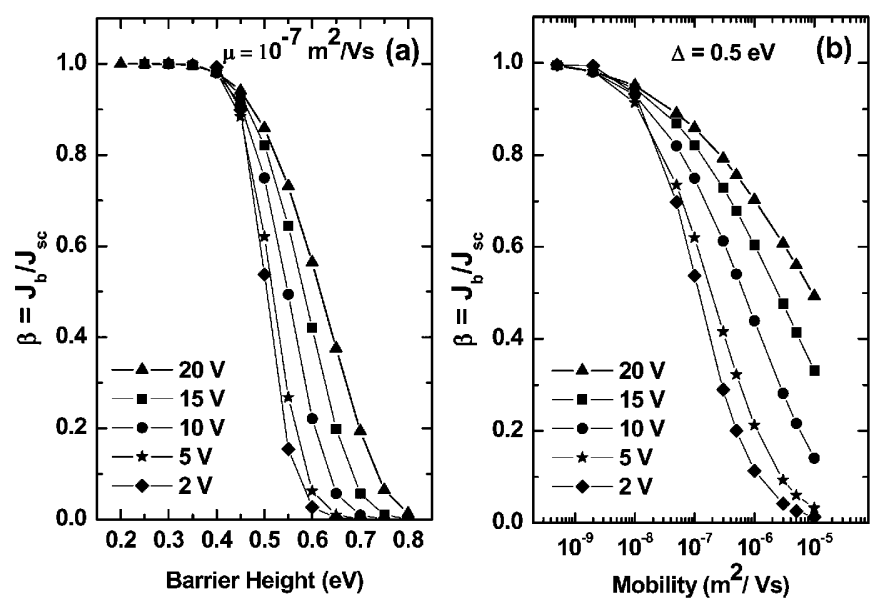

Fig. 5. (a) Dependence of the analytical function $\beta$ on the barrier height. (b) Mobility). The following material parameters are used: attempt-to-jump frequency $\nu_{0}=4.75 \times 10^{13} \mathrm{~s}^{-1}$, inverse localization radius $\gamma=5 \times 10^{9} \mathrm{~m}^{-1}$, DOS width $\sigma=50 \mathrm{~m} \mathrm{eV}$, site density $N_{t}=5 \times 10^{20} \mathrm{~cm}^{-3}$, intersite distance $\mathrm{a}=1 \mathrm{~nm}$, and dielectric constant $\varepsilon=4$.

remaining parameters are in the usual order for PLEDs [7], [10], and their physical discussion is considered beyond the purpose of this paper.

In order to demonstrate the validity of this model, diodes of the same material, and then, with the same set of parameters, but with different thicknesses have been fabricated, characterized and compared with the model predictions. Experimental I-V curves (dotted lines) are plotted in Fig. 4. Solid lines in this figure show theoretical simulations using the corresponding thicknesses, measured by profilometry. Assuming measurement uncertainties and disregarding nonideal behavior such as leakage currents, the agreement between theory and experiment is reasonably good.

According to [1], current through a typical organic diode should be bulk limited, when barrier heights are lower than $0.2 \mathrm{eV}$. In that case, the current across the device will be max- imum and would obey Mott-Gurney's law (10). If the contact is clearly non-ohmic (i.e., barrier heights greater that $0.3 \mathrm{eV}$ ), injection limits the maximum current given by (10), but still, even if little conduction occurs in the organic device, it should follow the space-charge law in (9). By applying the model proposed in this paper, it is possible to determine the contribution of space charge in any limiting regime, bulk or injection, and no simplifications need to be done.

The inset of Fig. 3 shows the dependence of the $\beta$ function on bias for both PLEDs. In both cases, due to the high barrier for carrier injection, $\beta$ remains close to 0 , as expected for injectionlimited conduction. Moreover, a gradual increase of $\beta$ for both diodes is observed for higher voltages, confirming that space charge effect becomes important with increasing bias. As one would also expect, the MDMO:PPV-based PLED will enter before in the bulk-limited regime, due to its lower barrier. This is reflected in a higher value for $\beta$. In this way, the factor $\beta$ enables us to quantify the amount of injection or space-charge effect.

Theoretical predictions about the conduction regime for diodes with other barriers and carrier mobilities are shown in Fig. 5. For several fixed values of $V_{b}$ and their corresponding $J_{b}$, Fig. 5 shows the dependence of $\beta$ on the barrier height (a) and mobilitiy (b) for a typical active layer thickness of $100 \mathrm{~nm}$. When $\Delta<0.4 \mathrm{eV}$, Fig. 5(a) shows that current across the diode equals the maximum current given by (10), so $\beta \approx 1$, and therefore, conduction is bulk limited. As barrier height increases, the current moves from a bulk-limited to an injection-limited regime. This regime shift occurs faster at lower bias. For intermediate barriers, $0.5<\Delta<0.6$, the conduction regime depends on the applied voltage. Further increasing $\Delta$, the injection limiting regime, $\beta \approx 0$, becomes dominant.

The transition from bulk to injection-limited conduction occurs at lower $\Delta$ values when dealing with higher mobilities. Fig. 5(b) illustrates that mobility also plays an important role in determining the limiting conduction mechanism. As mobility increases, the accumulated space-charge is reduced, and the field at the interface approaches the total applied external field $\left(E_{0} \approx V_{b} / t\right)$. In other words, the total current $J_{b}$ is smaller than that predicted by a pure space-charge mechanism (10), so that the injection mechanism becomes dominant. As mobility decreases, the field at the interface is a smaller fraction of $\mathrm{V}_{\mathrm{b}} / \mathrm{t}$, and the total current approaches that given by Mott-Gurney law. Fig. 5 indicates to what extent reducing interface barrier and mobility favours bulk limiting conduction.

In conclusion, we have proposed and tested a complete model for conduction in single carrier organic diodes in which both injection and bulk currents are simultaneously considered. Traditionally, the bulk regime is considered to exist in those structures with quasi-ohmic contact. The model confirms the belief, that space-charge current may also play an important role in structures with significant values of the barrier height $(0.3-0.7 \mathrm{eV})$, depending on sample thickness, applied bias, and carrier mobility. An analytical function, $\beta$, has been defined and used to quantify how close we are to the extreme simplifications, injection, and bulk limited conduction, using measurable quantities, such as current and external bias. 


\section{ACKNOWLEDGMENT}

The authors wish to acknowledge Instituto de Sistemas Optoelectrónicos y Microtecnología (ISOM, U.P.M) for their valuable help with profilometry measurements.

\section{REFERENCES}

[1] V. I. Arkhipov, H. von Seggern, and E. V. Emelianova, "Charge injection versus space-charge-limited current in organic light emitting diodes," Appt. Phys. Lett., vol. 83, pp. 5074-5076, 2003.

[2] M. A. Abkowitz, H. A. Mizes, and J. S. Facci, "Emission limited injection by thermally assisted tunnelling into a trap-free transport polymer," Appl. Phys. Lett., vol. 66, pp. 1288-1290, 1995.

[3] Y. Roichman, Y. Preezant, and N. Tessler, "Analysis and modelling of organic devices," Phys. Stat. Sol., vol. 201, pp. 1246-1262, 2004.

[4] J. Campbell Scott and G. G. Malliaras, "Charge injection and recombination at the metal-organic interface," Chem. Phys. Lett., vol. 299, pp. $115-119,1999$.

[5] P. S. Davids, I. H. Campbell, and D. L. Smith, "Device model for single carrier organic diodes," J. Appl. Phys., vol. 82, pp. 6319-6325, 1997.

[6] H. Bässler, "Charge transport in disordered organic photoconductors," Phys. Stat. Sol. B, vol. 175, pp. 15-56, 1993.

[7] V. I. Arkhipov, E. V. Emelianova, Y. H. Tak, and H. Bässler, "Charge injection into light-emitting diodes: Theory and experiment," $J$. Appl. Phys., vol. 84, pp. 848-856, 1998.

[8] T. van Woudenberg, P. W. Blom, M. C. J. M. Vissenberg, and J. N. Huiberts, "Temperature dependence of the charge injection in poly-dialkoxy-pphenilene vinylene," Appl. Phys. Lett., vol. 79, pp. 1697-1699, 2001.

[9] N. F. Mott and R. W. Gurney, Electronic Process in Ionic Crystals, 2nd ed. London, U.K.: Oxford Univ. Press, 1948.

[10] V. I. Arkhipov, E. V. Emelianova, and G. J. Adriaenssens, "Effective transport energy versus the energy of most probable jumps in disordered hopping systems," Phys. Rev. B, vol. 64, pp. 125125 (6), 2001.

[11] V. I. Arkhipov, P. Heremans, E. V. Emelianova, and G. J. Adriaenssens, "Space-charge-limited currents in materials with gaussian energy distributions of localized states," Appl. Phys. Lett, vol. 79, p. 4154, 2001.

[12] V. I. Arkhipov, E. V. Emelianova, and H. Bässler, "Equilibrium carrier mobility in disordered hopping systems," Philos. Mag. B, vol. 81, p. 985 , 2001.

[13] V. I. Arkhipov, H. von Seggern, and E. V. Emelianova, "Charge injection versus space-charge-limited current in organic light emitting diodes," Appl. Phys. Lett., vol. 83, p. 5074, 2003.

[14] R. Mallavia, F. Montilla, I. Pastor, P. Velasquez, B. Arredondo, A. L. Alvarez, and C. R. Mateo, "Characterization and side chain manipulation in violet-blue poly-[(9,9-dialkylfluoren-2,7-diyl)-alt-co-(benzen1,4-diyl) backbones," Macromolecules, vol. 38, p. 3185, 2005.

[15] T. Bäck, U. Hammel, and H.-P. Schwefel, "Evolutionary computation: Comments on the history and current state," IEEE Trans. Evol. Comput., vol. 1, no. 1, pp. 3-17, Apr. 1997.

[16] B. Arredondo, B. Romero, A. L. Alvarez, A. Gutiérrez-Llorente, X. Quintana, R. Mallavia, R. Vergaz, F. Montilla, J. M. Sánchez-Pena, and J. M Otón,, "Determination of hole mobilities in new blue emitting organic diodes by means of impedance spectroscopy," J. Soc. Inf. Displays, vol. 38, no. 1, pp. 841-844, 2007.

[17] H. Frohne, C. R. McNeill, G. G. Wallace, and P. C. Dastoor, "Enhancement of polymer electronics via surface states on highly doped polymeric anodes," J. Phys. D: Appl. Phys., vol. 37, p. 165, 2004.

[18] W. F. Pasveer, J. Cottaar, C. Tanase, R. Coehoorn, P. A. Bobbert, P. W. M. Blom, D. M. de Leeuw, and M. A. J. Michels, "Unified description of charge-carrier mobilities in disordered semiconducting polymers," Phys. Rev. Lett., vol. 94, pp. 206601-206611, 2005.

[19] J. Bisquert, J. M. Montero, H. J. Bolink, E. M. Barea, and G. GarciaBelmonte, "Thickness scaling of space-charge-limited currents in organic layers with field- or density-dependent mobility," Phys. Stat. Sol. A, vol. 203, pp. 3762-3767, 2006.

[20] C. Tanasse, E. J. Meijer, P. W. M. Blom, and D. M. de Leeuw, "Unification of the hole transport in polymeric field-effect-transistors and light-emitting diodes," Phys. Rev. Lett., vol. 91, pp. 216601-216611, 2003.

[21] C. Vijila, A. Pivrikas, H. Chun, C. Zhikuan, R. Österbacka, and C. S. Jin, "A study of charge transport in a novel electroluminescent poly(phenylene vinylene-co-uorenylene vinylene)based p-conjugated polymer," Organic Electron., vol. 8-13, p. 8, 2007.

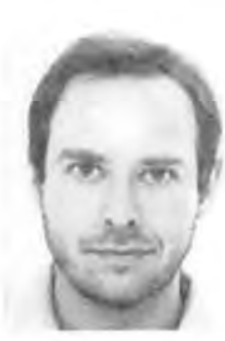

Ángel Luis Alvarez (A'03-M'04) received the M.S. degree in physics (materials science) from the Universidad Complutense, Madrid, in 1990, and the Ph.D. degree in spectroscopic characterization of optoelectronic devices from E.T.S.I Telecomunicación (the Universidad Politécnica de Madrid), Madrid, Spain, in 1996.

In 1998, he was an Associate Professor at the Universidad Miguel Hernández (Elche), Alicante, Spain, where he joined the Institute of Bioengineering in 2001. During this period, he joined a group to develop organic materials and applications. In October 2003, he joined the Universidad Rey Juan Carlos, Madrid, where he founded the Organic Optoelectrics Group. His current research interests include organic device modeling as well as novel lithographic techniques for organic displays with specific applications.

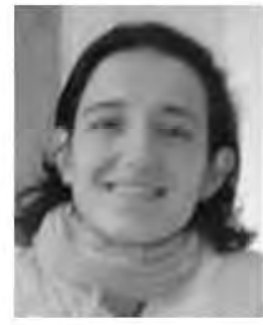

Belén Arredondo was born in Madrid, Spain, in 1975. She received the M.S. degree in physics (electronics) from the University Complutense, Madrid, in 1999. She is currently working toward the Ph.D. degree in polymer light at the Organic Optoelectronics Group, University Rey Juan Carlos, Madrid.

She worked in the Electromagnetics Division of the European Space Research and Technology Centre (ESTEC), Noordwijk, The Netherlands, as a Research Fellow for two years.

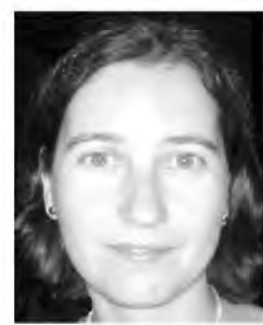

Beatriz Romero was born in Guadalajara, Spain, in 1970. She received the M.S. degree in physics (electronics) from the Universidad Complutense de Madrid, Madrid, Spain, in 1993, and the Ph.D. degree from the Universidad Politécnica de Madrid, Madrid, Spain, in 1998

In 1999, she joined the Universidad Rey Juan Carlos, Madrid, where she is an Associate Professor in the Organic Optoelectronics Group. Her current research interests include polymeric light-emitting diodes, fabrication, characterization, and modeling, as well as organic displays.

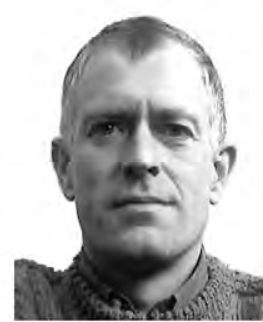

Xabier Quintana received the $\mathrm{Ph}$.D. degree in telecommunication engineering from the Universidad Politécnica, Madrid, Spain, in 1996.

$\mathrm{He}$ is currently an Associate Professor in the Department of Photonic Technology, School of Telecom Engineers, Universidad Politécnica de Madrid, where he teaches courses on charge coupled device (CCD) and $\mathrm{CMOS}$ detectors, fiber optics, and optical communications. His current research interests include displays and photonic devices based on liquid crystals and on organic light-emitting devices (LEDs). He has coauthored over 40 scientific papers and presented over 90 communications to scientific conferences.

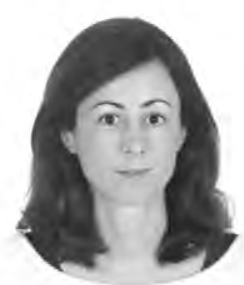

Araceli Gutiérrez-Llorente received the five-year degree in physics and the Ph.D. degree in applied physics from the Universidad Autónoma de Madrid, Madrid, Spain, in 1994 and 1999, respectively.

Dr. Gutiérrez-Llorente is currently a "Ramón y Cajal" Researcher at "Universidad Rey Juan Carlos," Madrid. Her current research interests include organic electronics. 


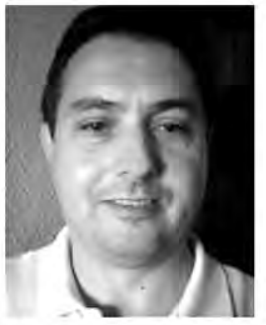

Ricardo Mallavia received his early education of chemistry from the Universidad Autónoma de Madrid, Madrid, Spain (speciality in organic chemistry), and the Ph.D. degree in chemistry of polymerization photo-initiators from Consejo Superior de Investigaciones Científicas (C.S.I.C.), Madrid, in 1994, where he was engaged in research in association with Profs. Francisco Amat and Roberto Sastre .

From 1994, he worked in the pharmaceutical industry, for two years, as a Quality Control Director of manufactured products. He returned as an Associate Professor at the Universidad Miguel Hernandez (Elche), Orihuela, Spain, until 2001. During this period, he focused on the development, analysis, and optimization of holographic materials in the field of polymers. In March 2002, he joined the Institute of Molecular and Cellular Biology to start independent research incorporated in a prestigious Spanish program, "Ramon y Cajal." His current research interests include the synthesis and characterization of functional conjugated polymers and the modulation of properties to be used in different applications: from polymer light-emitting diodes (PLEDs) to specific sensors.

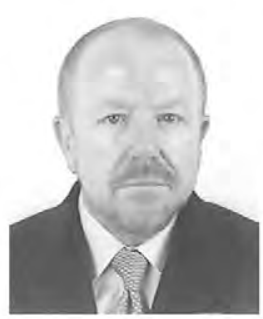

José Manuel Otón received the Ph.D. degree in chemistry from the Universidad Complutense, Madrid, Spain, in 1976.

$\mathrm{He}$ is a Full Professor in the Department of Photonic Technology, School de Telecom Engineers, Universidad Politécnica de Madrid, Madrid, Spain, where he teaches courses on lasers, fiber optics, and optical communications.

Mr. Otón current research interests include displays and photonic devices based on liquid crystals and on organic light-emitting devices (LEDs). He has coauthored over 100 scientific papers and presented over 150 communications to scientific conferences. 